\title{
ON SOME INEQUALITIES IN NORMED LINEAR SPACES
}

\author{
S. S. DRAGOMIR
}

\begin{abstract}
Upper and lower bounds for the norm of a linear combination of vectors are given. Applications in obtaining various inequalities for the quantities $\|x /\| x \|-$ $y /\|y\| \|$ and $\|x /\| y\|-y /\| x\|\| \|$, where $x$ and $y$ are nonzero vectors, that are related to the Massera-Schäffer and the Dunkl-Williams inequalities are also provided. Some bounds for the unweighted Čebyšev functional are given as well.
\end{abstract}

\section{Introduction}

In [9], L. Maligranda has obtained the following interesting inequality for two nonzero vectors $x, y$ in a real or complex normed linear space $(X,\|\cdot\|)$ :

$$
\left\|\frac{x}{\|x\|}-\frac{y}{\|y\|}\right\| \leq \frac{\|x-y\|+|\|x\|-\|y\||}{\max \{\|x\|,\|y\|\}} .
$$

Notice that, this inequality provides a refinement for the celebrated Massera-Schäffer inequality [10]:

$$
\left\|\frac{x}{\|x\|}-\frac{y}{\|y\|}\right\| \leq \frac{2\|x-y\|}{\max \|x\|,\|y\|\}},
$$

which, in its turn, is a refinement of the Dunkl-Williams inequality [7]

$$
\left\|\frac{x}{\|x\|}-\frac{y}{\|y\|}\right\| \leq \frac{4\|x-y\|}{\|x\|+\|y\|}
$$

More recently, in order to provide a lower bound for the quantity $\|x /\| x\|-y /\| y\|\|, \mathrm{P}$. R. Mercer obtained in [11] the following result as well:

$$
\frac{\|x-y\|-|\|x\|-\|y\||}{\min \{\|x\|,\|y\|\}} \leq\left\|\frac{x}{\|x\|}-\frac{y}{\|y\|}\right\| .
$$

In an effort to generalise the above results for $n$-vectors, J. Pečarić and R. Rajić have obtained in [13] the following double inequality:

$$
\max _{k \in\{1, \ldots, n\}}\left\{\frac{1}{\left\|x_{k}\right\|}\left[\left\|\sum_{j=1}^{n} x_{j}\right\|-\sum_{j=1}^{n}\left|\left\|x_{j}\right\|-\left\|x_{k}\right\|\right|\right]\right\}
$$

Received August 6, 2008.

2000 Mathematics Subject Classification. 26D15, 46B05.

Key words and phrases. Normed linear spaces, Triangle inequality, Massera-Schäffer inequality, Dunkl-Williams inequality, Čebyšev functional. 


$$
\left.\leq\left\|\sum_{j=1}^{n} \frac{x_{j}}{\left\|x_{j}\right\|}\right\| \leq \min _{k \in\{1, \ldots, n\}}\left\{\frac{1}{\left\|x_{k}\right\|}\left[\left\|\sum_{j=1}^{n} x_{j}\right\|+\sum_{j=1}^{n} \mid\left\|x_{j}\right\|-\left\|x_{k}\right\|\right]\right]\right\},
$$

for any $x_{j} \in X \backslash\{0\}$, where $j \in\{1, \ldots, n\}$ and observed that, for $n=2, x_{1}=x$ and $x_{2}=-y,(1.5)$ reduces to the Maligranda $\&$ Mercer inequalities outlined above. They also remarked that, the following refinement of the generalised triangle inequality obtained by M. Kato et al. in [8]

$$
\begin{aligned}
& \min _{k \in\{1, \ldots, n\}}\left\{\left\|x_{k}\right\|\right\}\left[n-\left\|\sum_{j=1}^{n} \frac{x_{j}}{\left\|x_{j}\right\|}\right\|\right] \\
& \leq \sum_{j=1}^{n}\left\|x_{j}\right\|-\left\|\sum_{j=1}^{n} x_{j}\right\| \\
& \leq \max _{k \in\{1, \ldots, n\}}\left\{\left\|x_{k}\right\|\right\}\left[n-\left\|\sum_{j=1}^{n} \frac{x_{j}}{\left\|x_{j}\right\|}\right\|\right]
\end{aligned}
$$

can be deduced from (1.5) as well.

We should remark that (1.6) can be also obtained as a particular case from the author's recent result established in [1]

$$
\begin{aligned}
& \max _{1 \leq j \leq n}\left\{\left\|x_{j}\right\|\right\}\left[\sum_{j=1}^{n}\left\|x_{j}\right\|^{p-1}-\left\|\sum_{j=1}^{n} \frac{x_{j}}{\left\|x_{j}\right\|}\right\|^{p}\right] \\
& \geq \sum_{j=1}^{n}\left\|x_{j}\right\|^{p}-n^{1-p}\left\|\sum_{j=1}^{n} x_{j}\right\|^{p} \\
& \geq \min _{1 \leq j \leq n}\left\{\left\|x_{j}\right\|\right\}\left[\sum_{j=1}^{n}\left\|x_{j}\right\|^{p-1}-\left\|\sum_{j=1}^{n} \frac{x_{j}}{\left\|x_{j}\right\|}\right\|^{p}\right],
\end{aligned}
$$

where $p \geq 1$ and $n \geq 2$.

Notice that, in [1], a more general result for convex functions has been obtained as well.

Motivated by the above results, we establish in this paper some upper and lower bounds for the more general quantity $\left\|\sum_{j=1}^{n} \alpha_{j} x_{j}\right\|$ where $\alpha_{j}, j \in\{1, \ldots, n\}$ are scalars in $\mathbb{K}(\mathbb{K}=\mathbb{C}, \mathbb{R})$ and $x_{j}, j \in\{1, \ldots, n\}$ are vectors in the normed linear space. For $\alpha_{j}=\left\|x_{j}\right\|$ with $x_{j} \in X \backslash\{0\} . j \in\{1, \ldots, n\}$ we obtain a result which is similar to (1.5). For the case of two vectors we recapture Maligranda's result (1.1) and provide various inequalities for the dual expression $x /\|y\|-y /\|x\| \|$ with $x, y \in X \backslash\{0\}$. Some bounds for the unweighted Čebyšev functional are given as well.

\section{Inequalities for $n$-Vectors}

The following result may be stated. 
Theorem 1. If $x_{k} \in X$ and $\alpha_{k} \in \mathbb{K}, k \in\{1, \ldots, n\}$, then

$$
\begin{aligned}
& \max _{k \in\{1, \ldots, n\}}\left\{\left|\sum_{j=1}^{n} \alpha_{j}\right|\left\|x_{k}\right\|-\sum_{j=1}^{n}\left|\alpha_{j}\right|\left\|x_{k}-x_{j}\right\|\right\} \\
& \leq\left\|\sum_{j=1}^{n} \alpha_{j} x_{j}\right\| \\
& \leq \min _{k \in\{1, \ldots, n\}}\left\{\left|\sum_{j=1}^{n} \alpha_{j}\right|\left\|x_{k}\right\|+\sum_{j=1}^{n}\left|\alpha_{j}\right|\left\|x_{k}-x_{j}\right\|\right\} .
\end{aligned}
$$

Proof. For any $k \in\{1, \ldots, n\}$, we have

$$
\sum_{j=1}^{n} \alpha_{j} x_{j}=\left(\sum_{j=1}^{n} \alpha_{j}\right) x_{k}+\sum_{j=1}^{n} \alpha_{j}\left(x_{j}-x_{k}\right) .
$$

Taking the norm in (2.2) and using the triangle inequality we have successively:

$$
\begin{aligned}
\left\|\sum_{j=1}^{n} \alpha_{j} x_{j}\right\| & \leq\left\|\left(\sum_{j=1}^{n} \alpha_{j}\right) x_{k}\right\|+\left\|\sum_{j=1}^{n} \alpha_{j}\left(x_{j}-x_{k}\right)\right\| \\
& \leq\left|\sum_{j=1}^{n} \alpha_{j}\right|\left\|x_{k}\right\|+\sum_{j=1}^{n}\left|\alpha_{j}\right|\left\|x_{k}-x_{j}\right\|
\end{aligned}
$$

for any $k \in\{1, \ldots, n\}$.

Taking the minimum over $k$ in (2.3) we deduce the second inequality in (2.1).

From (2.2) we also have

$$
\sum_{j=1}^{n} \alpha_{j} x_{j}=\left(\sum_{j=1}^{n} \alpha_{j}\right) x_{k}-\sum_{j=1}^{n} \alpha_{j}\left(x_{k}-x_{j}\right) .
$$

Taking in this equality the norm and using the continuity property of the norm, we have

$$
\begin{aligned}
\left\|\sum_{j=1}^{n} \alpha_{j} x_{j}\right\| & \geq\left\|\left(\sum_{j=1}^{n} \alpha_{j}\right) x_{k}\right\|-\left\|\sum_{j=1}^{n} \alpha_{j}\left(x_{k}-x_{j}\right)\right\| \mid \\
& \geq\left\|\left(\sum_{j=1}^{n} \alpha_{j}\right) x_{k}\right\|-\left\|\sum_{j=1}^{n} \alpha_{j}\left(x_{k}-x_{j}\right)\right\| \\
& \geq\left|\sum_{j=1}^{n} \alpha_{j}\right|\left\|x_{k}\right\|-\sum_{j=1}^{n}\left|\alpha_{j}\right|\left\|x_{k}-x_{j}\right\|,
\end{aligned}
$$

for each $k \in\{1, \ldots, n\}$. 
Taking the maximum in (2.4) we deduce the first part of (2.1).

Remark 1. If there exists an $r>0$ such that $\left\|x_{j}-x_{k}\right\| \leq r\left\|x_{k}\right\|$ for each $j, k \in$ $\{1, \ldots, n\}$, then we get from the second inequality in (2.1) that

$$
\left\|\sum_{j=1}^{n} \alpha_{j} x_{j}\right\| \leq \min _{k \in\{1, \ldots, n\}}\left\|x_{k}\right\|\left[\left|\sum_{j=1}^{n} \alpha_{j}\right|+r \sum_{j=1}^{n}\left|\alpha_{j}\right|\right] .
$$

Moreover, if $\alpha_{j} \in \mathbb{K}, j \in\{1, \ldots, n\}$ are such that

$$
\left|\sum_{j=1}^{n} \alpha_{j}\right| \geq r \sum_{j=1}^{n}\left|\alpha_{j}\right|
$$

(and in this case $r$ should be in $(0,1))$ then the opposite inequality

$$
\max _{k \in\{1, \ldots, n\}}\left\|x_{k}\right\|\left[\left|\sum_{j=1}^{n} \alpha_{j}\right|+r \sum_{j=1}^{n}\left|\alpha_{j}\right|\right] \leq\left\|\sum_{j=1}^{n} \alpha_{j} x_{j}\right\|
$$

also holds.

Corollary 1. For any nonzero vectors $x_{k} \in X, k \in\{1, \ldots, n\}$, we have the inequalities:

$$
\begin{aligned}
& \max _{k \in\{1, \ldots, n\}}\left\{\left\|x_{k}\right\| \sum_{j=1}^{n} \frac{1}{\left\|x_{j}\right\|}-\sum_{j=1}^{n} \frac{\left\|x_{k}-x_{j}\right\|}{\left\|x_{j}\right\|}\right\} \\
& \leq \sum_{j=1}^{n}\left\|\frac{x_{j}}{\left\|x_{j}\right\|}\right\| \\
& \leq \min _{k \in\{1, \ldots, n\}}\left\{\left\|x_{k}\right\| \sum_{j=1}^{n} \frac{1}{\left\|x_{j}\right\|}+\sum_{j=1}^{n} \frac{\left\|x_{k}-x_{j}\right\|}{\left\|x_{j}\right\|}\right\}
\end{aligned}
$$

and

$$
\begin{aligned}
& \max _{k \in\{1, \ldots, n\}}\left\{\left\|x_{k}\right\| \sum_{j=1}^{n}\left\|x_{j}\right\|-\sum_{j=1}^{n}\left\|x_{j}\right\|\left\|x_{k}-x_{j}\right\|\right\} \\
& \leq\left\|\sum_{j=1}^{n}\right\| x_{j}\left\|x_{j}\right\| \\
& \leq \min _{k \in\{1, \ldots, n\}}\left\{\left\|x_{k}\right\| \sum_{j=1}^{n}\left\|x_{j}\right\|+\sum_{j=1}^{n}\left\|x_{j}\right\|\left\|x_{j}-x_{k}\right\|\right\} .
\end{aligned}
$$




\section{Inequalities for Two Vectors}

The case for two vectors is of interest due to the fact that some similar inequalities obtained in the past by several authors have been applied in investigating various problems in the Geometry of Banach spaces, including the characterization problem of strict convexity and the characterization of inner product spaces in the larger class of normed spaces.

Lemma 1. For any $\alpha, \beta \in \mathbb{K}$ and $x, y \in X$ we have

$$
\begin{aligned}
& \frac{1}{2}[(\|x\|+\|y\|)|\alpha+\beta|-(|\alpha|+|\beta|)\|x-y\|] \\
& \quad+\frac{1}{2}|| \alpha+\beta|(\|x\|-\|y\|)+(|\alpha|-|\beta|)\|x-y\|| \\
& \leq\|\alpha x+\beta y\| \\
& \leq \frac{1}{2}[|\alpha+\beta|(\|x\|+\|y\|)+(|\alpha|+|\beta|)\|x-y\|] \\
& \quad-\frac{1}{2}|| \alpha+\beta|(\|x\|-\|y\|)-(|\alpha|-|\beta|)\|x-y\|| .
\end{aligned}
$$

Proof. If we choose in Theorem $1, \alpha_{1}=\alpha, \alpha_{2}=\beta, x_{1}=x$ and $x_{2}=y$, then we get

$$
\begin{aligned}
\max & \{\|x\||\alpha+\beta|-|\beta|\|x-y\|,\|y\||\alpha+\beta|-|\alpha|\|x-y\|\} \\
& \leq\|\alpha x+\beta y\| \\
& \leq \min \{\|x\||\alpha+\beta|+|\beta|\|x-y\|,\|y\||\alpha+\beta|+|\alpha|\|x-y\|\} .
\end{aligned}
$$

Utilising the properties for real numbers

$$
\max \{a, b\}=\frac{1}{2}[a+b+|a-b|] \text { and } \min \{a, b\}=\frac{1}{2}[a+b-|a-b|], \quad a, b \in \mathbb{R}
$$

we have:

$$
\begin{aligned}
\max & \{\|x\||\alpha+\beta|-|\beta|\|x-y\|,\|y\||\alpha+\beta|-|\alpha|\|x-y\|\} \\
= & \frac{1}{2}[(\|x\|+\|y\|)|\alpha+\beta|-(|\alpha|+|\beta|)\|x-y\|] \\
& +\frac{1}{2}|| \alpha+\beta|(\|x\|-\|y\|)+(|\alpha|-|\beta|)\|x-y\||
\end{aligned}
$$

and

$$
\begin{gathered}
\min \{\|x\||\alpha+\beta|+|\beta|\|x-y\|,\|y\||\alpha+\beta|+|\alpha|\|x-y\|\} \\
=\frac{1}{2}[|\alpha+\beta|(\|x\|+\|y\|)+(|\alpha|+|\beta|)\|x-y\|]
\end{gathered}
$$




$$
-\frac{1}{2}|\alpha+\beta|(\|x\|-\|y\|)-(|\alpha|-|\beta|)\|x-y\| \mid,
$$

which, by (3.2) produces the desired result (3.1).

The following particular cases are of interest:

Corollary 2. If $\alpha, \beta \in \mathbb{K}$ with $|\alpha|=|\beta|=1$, then

$$
\left|\|\alpha x+\beta y\|-\frac{1}{2}(\|x\|+\|y\|)\right| \alpha+\beta|| \leq\|x-y\|-\frac{1}{2}|\alpha+\beta||\|x\|-\|y\||,
$$

for any $x, y \in X$.

Corollary 3. If $x, y \in X$ with $\|x\|=\|y\|=1$, then

$$
|\|\alpha x+\beta y\|-| \alpha+\beta|| \leq \max \{|\alpha|,|\beta|\}\|x-y\|,
$$

for any $\alpha, \beta \in \mathbb{K}$.

Corollary 4. For any two nonzero vectors $x, y \in X$ we have:

$$
\left\|\frac{x}{\|x\|}-\frac{y}{\|y\|}\right\| \leq \frac{\|x-y\|+|\|x\|-\|y\||}{\max \{\|x\|,\|y\|\}} .
$$
get

Proof. We choose in the second inequality from (3.2) $\alpha=\frac{1}{\|x\|}$ and $\beta=\frac{-1}{\|y\|}$, then we

$$
\begin{aligned}
& \left\|\frac{x}{\|x\|}-\frac{y}{\|y\|}\right\| \\
& \quad \leq \min \left\{\frac{\|x\||\|x\|-\|y\||}{\|x\|\|y\|}+\frac{\|x-y\|}{\|y\|}, \frac{\|y\||\|x\|-\|y\||}{\|x\|\|y\|}+\frac{\|x-y\|}{\|x\|}\right\} \\
& =[\|x-y\|+|\|x\|-\|y\||] \min \left\{\frac{1}{\|x\|}, \frac{1}{\|y\|}\right\} \\
& \quad=\frac{\|x-y\|+|\|x\|-\|y\||}{\max \{\|x\|,\|y\|\}}
\end{aligned}
$$

and the inequality (3.5) is proved.

Remark 2. The inequality (3.5) has been firstly obtained by L. Maligranda in [9] on utilising a different approach.

Corollary 5. For any two nonzero vectors $x, y \in X$ we have the reverse of the triangle inequality

$$
(0 \leq)\|x\|+\|y\|-\|x+y\| \leq\left\|\frac{x}{\|x\|}-\frac{y}{\|y\|}\right\| \min \{\|x\|,\|y\|\} .
$$


Proof. If we write the first inequality in (3.2) for $-y$ and for $\alpha=\frac{1}{\|x\|}, \beta=\frac{1}{\|y\|}$, then we get

$$
\begin{aligned}
& \max \left\{\frac{\|x\|(\|x\|+\|y\|)}{\|x\|\|y\|}-\frac{\|x+y\|}{\|y\|}, \frac{\|y\|(\|x\|+\|y\|)}{\|x\|\|y\|}-\frac{\|x+y\|}{\|x\|}\right\} \\
& \quad \leq\left\|\frac{x}{\|x\|}-\frac{y}{\|y\|}\right\|,
\end{aligned}
$$

which is clearly equivalent to (3.7).

Remark 3. In [9], P. R. Mercer has obtained the following lower bound for the quantity $\left\|\frac{x}{\|x\|}-\frac{y}{\|y\|}\right\|$ :

$$
\frac{\|x-y\|-|\|x\|-\|y\||}{\min \{\|x\|,\|y\|\}} \leq\left\|\frac{x}{\|x\|}-\frac{y}{\|y\|}\right\|
$$

for any $x, y \in X \backslash\{0\}$.

In order to compare the lower bounds provided by (3.7) and (3.8) consider $B_{1}, B_{2}$ : $X^{2} \rightarrow \mathbb{R}$

$$
B_{1}(x, y):=\|x\|+\|y\|-\|x+y\|
$$

and

$$
B_{2}(x, y):=\|x-y\|-|\|x\|-\|y\||
$$

Now, we observe that

$$
\begin{aligned}
B_{2}(x, y)-B_{1}(x, y) & =\|x-y\|+\|x+y\|-[\|x\|+\|y\|+\|\| x\|-\| y \| \mid] \\
& =\|x-y\|+\|x+y\|-2 \max \{\|x\|,\|y\|\} \geq 0
\end{aligned}
$$

for any $x, y \in X$. Therefore the Mercer result is better than (3.7) in providing a lower bound for the quantity $\left\|\frac{x}{\|x\|}-\frac{y}{\|y\|}\right\|$.

In the following we consider the dual problem, namely the problem of finding upper and lower bounds for the quantity

$$
\left\|\frac{x}{\|y\|}-\frac{y}{\|x\|}\right\|
$$

where $x, y \in X \backslash\{0\}$.

The first result that provides a lower bound is incorporated in

Theorem 2. For any two nonzero vectors $x, y \in X$ we have:

$$
0 \leq \frac{\|x\|+\|y\|}{\min \{\|x\|,\|y\|\}}-\frac{\|x+y\|}{\max \{\|x\|,\|y\|\}} \leq\left\|\frac{x}{\|y\|}-\frac{y}{\|x\|}\right\| .
$$


Proof. Taking $\alpha=\frac{1}{\|y\|}$ and $\beta=\frac{1}{\|x\|}$ in the left side of the inequality (3.2) we have

$$
\begin{aligned}
\max \{ & \left.\frac{\|x\|(\|x\|+\|y\|)}{\|x\|\|y\|}-\frac{\|x-y\|}{\|x\|}, \frac{\|y\|(\|x\|+\|y\|)}{\|x\|\|y\|}-\frac{\|x-y\|}{\|y\|}\right\} \\
= & \frac{1}{2}\left[(\|x\|+\|y\|) \cdot \frac{\|x\|+\|y\|}{\|x\|\|y\|}-\frac{\|x-y\|(\|x\|+\|y\|)}{\|x\|\|y\|}\right] \\
& +\frac{1}{2}\left|\frac{(\|x\|+\|y\|)(\|x\|-\|y\|)}{\|x\|\|y\|}+\frac{\|x-y\|(\|x\|-\|y\|)}{\|x\|\|y\|}\right| \\
= & \frac{1}{2} \cdot \frac{(\|x\|+\|y\|)(\|x\|+\|y\|)}{\|x\|\|y\|}-\frac{1}{2} \cdot \frac{\|x-y\|(\|x\|+\|y\|)}{\|x\|\|y\|} \\
& +\frac{1}{2} \cdot \frac{\|x\|-\|y\| \mid}{\|x\|\|y\|}(\|x\|+\|y\|)+\frac{1}{2} \cdot \frac{\|x\|-\|y\| \mid}{\|x\|\|y\|}\|x-y\| \\
= & \frac{1}{2} \cdot \frac{\|x\|+\|y\|}{\|x\|\|y\|}(\|x\|+\|y\|+\mid\|x\|-\|y\|) \\
& -\frac{1}{2} \cdot \frac{\|x-y\|}{\|x\|\|y\|}(\|x\|+\|y\|-\mid\|x\|-\|y\|) \\
= & (\|x\|+\|y\|) \max \left\{\frac{1}{\|x\|}, \frac{1}{\|y\|}\right\}-\|x-y\| \min \left\{\frac{1}{\|x\|}, \frac{1}{\|y\|}\right\} \\
= & \frac{\|x\|+\|y\|}{\min \{\|x\|,\|y\|\}}-\frac{\|x-y\|}{\max \{\|x\|,\|y\|\}} .
\end{aligned}
$$

Then, by the first inequality in (3.2) we get

$$
\frac{\|x\|+\|y\|}{\min \{\|x\|,\|y\|\}}-\frac{\|x-y\|}{\max \{\|x\|,\|y\|\}} \leq\left\|\frac{x}{\|x\|}+\frac{y}{\|y\|}\right\|
$$

which clearly implies (3.9).

Theorem 3. For any two nonzero vectors $x, y \in X$ we have

$$
\left\|\frac{x}{\|y\|}-\frac{y}{\|x\|}\right\| \leq \frac{|\|x\|-\|y\||}{\min \{\|x\|,\|y\|\}}+\frac{\|x-y\|}{\max \{\|x\|,\|y\|\}} .
$$

Proof. Taking $\alpha=\frac{1}{\|y\|}$ and $\beta=-\frac{1}{\|x\|}$ in the right side of (3.2) we have successively

$$
\begin{gathered}
\min \left\{\|x\| \frac{|\|x\|-\|y\||}{\|x\|\|y\|}+\frac{1}{\|x\|}\|x-y\|,\|y\| \cdot \frac{|\|x\|-\|y\||}{\|x\|\|y\|}+\frac{1}{\|y\|} \cdot\|x-y\|\right\} \\
=\frac{1}{2}\left[\frac{(\|x\|+\|y\|)|\|x\|-\|y\||}{\|x\|\|y\|}+\frac{\|x-y\|(\|x\|+\|y\|)}{\|x\|\|y\|}\right]
\end{gathered}
$$




$$
\begin{aligned}
& -\frac{1}{2}\left|\frac{(\|x\|-\|y\|)|\|x\|-\|y\||}{\|x\|\|y\|}-\frac{\|x-y\|(\|x\|-\|y\|)}{\|x\|\|y\|}\right| \\
= & \frac{1}{2} \frac{(\|x\|+\|y\|)|\|x\|-\|y\||}{\|x\|\|y\|}+\frac{1}{2} \frac{\|x-y\|(\|x\|+\|y\|)}{\|x\|\|y\|} \\
& -\frac{1}{2} \frac{|\|x\|-\|y\||}{\|x\|\|y\|}[\|x-y\|-|\|x\|-\|y\||] \\
= & \frac{1}{2} \frac{(\|x\|+\|y\|)|\|x\|-\|y\||}{\|x\|\|y\|}+\frac{1}{2} \frac{|\|x\|-\|y\|| \cdot|\|x\|-\|y\||}{\|x\|\|y\|} \\
& +\frac{1}{2} \frac{\|x-y\|(\|x\|+\|y\|)}{\|x\|\|y\|}-\frac{1}{2} \frac{\|x-y\||\|x\|-\|y\||}{\|x\|\|y\|} \\
= & \frac{1}{2} \frac{|\|x\|-\|y\||}{\|x\|\|y\|}[\|x\|+\|y\|+|\|x\|-\|y\||] \\
& \left.+\frac{1}{2} \frac{\|x-y\|}{\|x\|\|y\|}[\|x\|+\|y\|-\mid\|x\|-\|y\|]\right] \\
= & |\|x\|-\|y\|| \max \left\{\frac{1}{\|x\|}, \frac{1}{\|y\|}\right\}+\|x-y\| \min \left\{\frac{1}{\|x\|}, \frac{1}{\|y\|}\right\} \\
= & \frac{|\|x\|-\|y\||}{\min \{\|x\|,\|y\|\}}+\frac{\|x-y\|}{\max \{\|x\|,\|y\|\}},
\end{aligned}
$$

and by the second part of (3.2) we get the desired result (3.10).

\section{Bounds for the Čebyšev Functional}

For $\beta=\left(\beta_{1}, \ldots, \beta_{n}\right) \in \mathbb{K}^{n}$ and $\mathbf{y}=\left(y_{1}, \ldots, y_{n}\right) \in X^{n}$, we consider the unweighted Čebyšev functional defined by

$$
C_{n}(\beta, \mathbf{y}):=\frac{1}{n} \sum_{j=1}^{n} \beta_{j} y_{j}-\frac{1}{n} \sum_{j=1}^{n} \beta_{j} \cdot \frac{1}{n} \sum_{j=1}^{n} y_{j}
$$

We remark that this functional has been considered previously by the author and some bounds have been established. We recall here some simple results. 
With the above assumptions for $X, \alpha$ and $\mathbf{y}$, we have

$$
\left\|C_{n}(\alpha, \mathbf{y})\right\| \leq\left\{\begin{array}{l}
\frac{1}{12}\left(n^{2}-1\right) \max _{j \in\{1, \ldots, n-1\}}\left|\Delta \alpha_{j}\right| \max _{j \in\{1, \ldots, n-1\}}\left\|\Delta y_{j}\right\|, \quad[6] \\
\frac{1}{2} \cdot\left(1-\frac{1}{n}\right) \sum_{j=1}^{n-1}\left|\Delta \alpha_{j}\right| \sum_{j=1}^{n-1}\left\|\Delta y_{j}\right\|,[3] \\
\frac{1}{6} \frac{n^{2}-1}{n}\left(\sum_{j=1}^{n-1}\left|\Delta \alpha_{j}\right|^{p}\right)^{1 / p}\left(\sum_{j=1}^{n-1}\left\|\Delta y_{j}\right\|^{q}\right)^{1 / q} \\
p>1, \frac{1}{p}+\frac{1}{q}=1,[2],
\end{array}\right.
$$

where $\Delta z_{j}=z_{j+1}-z_{j}$ is the forward difference. Here the constants $\frac{1}{12}, \frac{1}{2}$ and $\frac{1}{6}$ are best possible in the sense that they cannot be replaced by smaller quantities.

In [5] we also have established that

$$
\begin{aligned}
& \left\|C_{n}(\alpha, \mathbf{y})\right\|
\end{aligned}
$$

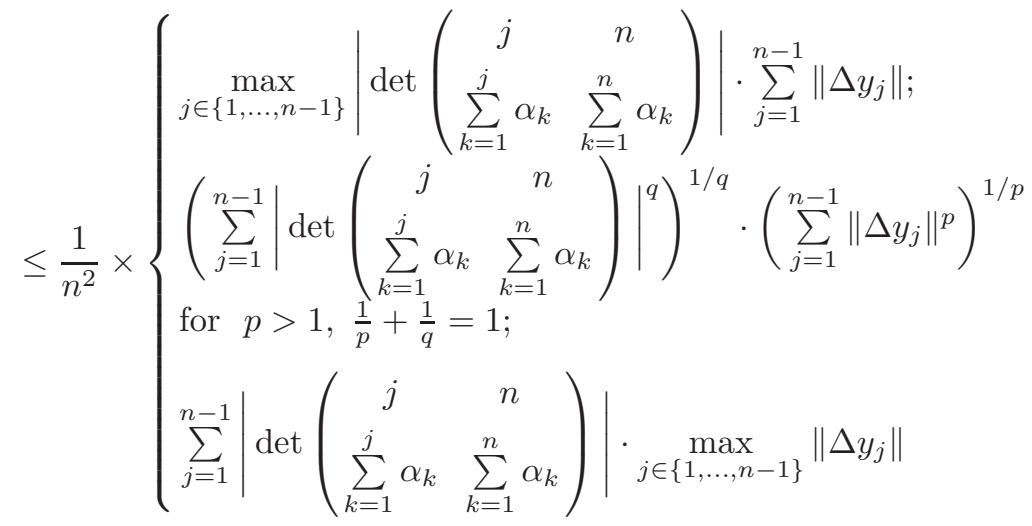

and

$$
\begin{aligned}
& \left\|C_{n}(\alpha, \mathbf{y})\right\| \\
& \leq \frac{1}{n} \times\left\{\begin{array}{l}
\max _{j \in\{1, \ldots, n-1\}}\left|\frac{1}{n} \sum_{k=1}^{n} \alpha_{k}-\frac{1}{j} \sum_{k=1}^{j} \alpha_{k}\right| \cdot \sum_{j=1}^{n-1} j\left\|\Delta y_{j}\right\| ; \\
\left(\sum_{j=1}^{n-1} j\left|\frac{1}{n} \sum_{k=1}^{n} \alpha_{k}-\frac{1}{j} \sum_{k=1}^{j} \alpha_{k}\right|^{q}\right)^{1 / q} \cdot\left(\sum_{j=1}^{n-1} j\left\|\Delta y_{j}\right\|^{p}\right)^{1 / p} \\
\text { for } p>1, \frac{1}{p}+\frac{1}{q}=1 ; \\
\sum_{j=1}^{n-1} j\left|\frac{1}{n} \sum_{k=1}^{n} \alpha_{k}-\frac{1}{j} \sum_{k=1}^{j} \alpha_{k}\right| \cdot \max _{j \in\{1, \ldots, n-1\} 1}\left\|\Delta y_{j}\right\| .
\end{array}\right.
\end{aligned}
$$

Finally, we recall the following result from [4]:

If there exists the complex numbers $a, A \in \mathbb{C}$ such that

$$
\operatorname{Re}\left[\left(A-\alpha_{j}\right)\left(\overline{\alpha_{j}}-\bar{a}\right)\right] \geq 0 \text { for each } j \in\{1, \ldots, n\}
$$


or, equivalently,

$$
\left|\alpha_{j}-\frac{a+A}{2}\right| \leq \frac{1}{2}|A-a| \text { for each } j \in\{1, \ldots, n\}
$$

then one has the inequality:

$$
\left\|C_{n}(\beta, \mathbf{y})\right\| \leq \frac{1}{2}|A-a| \cdot \frac{1}{n} \sum_{j=1}^{n}\left\|y_{j}-\frac{1}{n} \sum_{j=1}^{n} y_{j}\right\| .
$$

The constant $\frac{1}{2}$ in the right hand side of the inequality is best possible in the sense that it cannot be replaced by a smaller constant.

For many other results that hold for $n$-tuples $\beta$ and $\mathbf{y}$ of real numbers we recommend the chapters devoted to Grüss and Čebyšev inequalities from the books [12] and [14].

In the following we provide other upper and lower bounds for $\left\|C_{n}(\beta, \mathbf{y})\right\|$ :

Proposition 1. For any $\beta$ and $\mathbf{y}$ as above, we have:

$$
\begin{aligned}
\left\|C_{n}(\beta, \mathbf{y})\right\| \leq & \min _{k \in\{1, \ldots, n\}}\left\{\frac{1}{n} \sum_{j=1}^{n}\left|\beta_{j}-\frac{1}{n} \sum_{l=1}^{n} \beta_{l}\right|\left\|y_{j}-y_{k}\right\|\right\} \\
& \leq\left\{\begin{array}{l}
\min _{k \in\{1, \ldots, n\}}\left\{\max _{j \in\{1, \ldots, n\}}\left\{\left\|y_{j}-y_{k}\right\|\right\}\right\} \cdot \frac{1}{n} \sum_{j=1}^{n}\left|\beta_{j}-\frac{1}{n} \sum_{l=1}^{n} \beta_{l}\right|, \\
\min _{k \in\{1, \ldots, n\}}\left\{\left[\frac{1}{n} \sum_{j=1}^{n}\left\|y_{j}-y_{k}\right\|^{p}\right]^{\frac{1}{p}}\right\} \cdot\left[\frac{1}{n} \sum_{j=1}^{n}\left|\beta_{j}-\frac{1}{n} \sum_{l=1}^{n} \beta_{l}\right|^{q}\right]^{\frac{1}{q}}, \\
\min _{k \in\{1, \ldots, n\}}\left\{\frac{1}{n} \sum_{j=1}^{n}\left\|y_{j}-y_{k}\right\|\right\} \cdot \max _{j \in\{1, \ldots, n\}}\left\{\left|\beta_{j}-\frac{1}{n} \sum_{l=1}^{n} \beta_{l}\right|\right\} .
\end{array}\right.
\end{aligned}
$$

Proof. We observe that

$$
C_{n}(\beta, \mathbf{y})=\frac{1}{n} \sum_{j=1}^{n}\left(\beta_{j}-\frac{1}{n} \sum_{l=1}^{n} \beta_{l}\right) y_{j}
$$

Now, on applying the second inequality in Theorem 1 for $\alpha_{j}=\beta_{j}-\frac{1}{n} \sum_{l=1}^{n} \beta_{l}$ and $x_{j}=y_{j}$, we deduce the first part of (4.4). The second part is obvious by the Hölder inequality.

The following result can be stated as well:

Proposition 2. For any $\beta=\left(\beta_{1}, \ldots, \beta_{n}\right) \in \mathbb{K}^{n}$ and $\mathbf{y}=\left(y_{1}, \ldots, y_{n}\right) \in X^{n}$ we have the double inequality:

$$
\max _{k \in\{1, \ldots, n\}}\left\{\left|\frac{1}{n} \sum_{j=1}^{n} \beta_{j}-\gamma\right|\left\|y_{k}-\frac{1}{n} \sum_{l=1}^{n} y_{l}\right\|-\frac{1}{n} \sum_{j=1}^{n}\left|\beta_{j}-\gamma\right|\left\|y_{j}-y_{k}\right\|\right\}
$$




$$
\begin{aligned}
& \leq\left\|C_{n}(\beta, \mathbf{y})\right\| \\
& \leq \min _{k \in\{1, \ldots, n\}}\left\{\left|\frac{1}{n} \sum_{j=1}^{n} \beta_{j}-\delta\right|\left\|y_{k}-\frac{1}{n} \sum_{l=1}^{n} y_{l}\right\|+\frac{1}{n} \sum_{j=1}^{n}\left|\beta_{j}-\delta\right|\left\|y_{j}-y_{k}\right\|\right\},
\end{aligned}
$$

where $\gamma$ and $\delta$ can betaken arbitrary in $\mathbb{K}$.

Proof. Follows from Theorem 1 on noting that

$$
C_{n}(\beta, \mathbf{y})=\frac{1}{n} \sum_{j=1}^{n}\left(\beta_{j}-t\right)\left(y_{j}-\frac{1}{n} \sum_{l=1}^{n} y_{l}\right)
$$

for any $t \in \mathbb{K}$.

Remark 4. As a particular case of interest we can state the following result:

$$
\begin{aligned}
& \max _{k \in\{1, \ldots, n\}}\left\{\left|\frac{1}{n} \sum_{j=1}^{n} \beta_{j}\right|\left\|y_{k}-\frac{1}{n} \sum_{l=1}^{n} y_{l}\right\|-\frac{1}{n} \sum_{j=1}^{n}\left|\beta_{j}\right|\left\|y_{j}-y_{k}\right\|\right\} \\
& \leq\left\|C_{n}(\beta, \mathbf{y})\right\| \\
& \leq \min _{k \in\{1, \ldots, n\}}\left\{\left|\frac{1}{n} \sum_{j=1}^{n} \beta_{j}\right|\left\|y_{k}-\frac{1}{n} \sum_{l=1}^{n} y_{l}\right\|+\frac{1}{n} \sum_{j=1}^{n}\left|\beta_{j}\right|\left\|y_{j}-y_{k}\right\|\right\} .
\end{aligned}
$$

\section{References}

[1] S. S. Dragomir, Bounds for the normalised Jensen functional, Bull. Austral. Math. Soc., 74(2006), 471-478.

[2] S. S. Dragomir, Another Grüss type inequality for sequences of vectors in normed linear spaces and applications, J. Comput. Anal. Appl., 4(2002), 155-172.

[3] S. S. Dragomir, A Grüss type inequality for sequences of vectors in normed linear spaces and applications, Tamsui Oxf. J. Math. Sci., 20(2004), 143-159.

[4] S. S. Dragomir, Grüss type discrete inequalities in normed linear spaces, revisited, Nonlinear Funct. Anal. Appl., 9(2004), 577-591.

[5] S. S. Dragomir, Bounding the Čebyšev functional for sequences of vectors in normed linear spaces, Filomat, 18(2004), 15-26.

[6] S. S. Dragomir and G. L. Booth, Grüss-Lupaş type inequality and its applications for the estimation of p-moments of guessing mappings, Math. Commun. 5(2000), 117-126.

[7] C. F. Dunkl and K. S. Williams, A simple norm inequality, Amer. Math. Monthly, 71(1964), $53-54$.

[8] M. Kato, K.-S. Saito and T. Tamura, Sharp triangle inequality and its reverse in Banach spaces, Math. Inequal. \& Appl. 10(2007), 461-470.

[9] L. Maligranda, Simple norm inequalities, Amer. Math. Monthly, 113(2006), 256-260.

[10] J. L. Massera and J. J. Schäffer, Linear differential equations and functional analysis (I), Ann. of Math., 67(1958), 517-573. 
[11] P.R. Mercer, The Dunkl-Williams inequality in an inner product space, Math. Inequal. \& Appl. 10(2007), 447-450.

[12] D. S. Mitrinović, J. Pečarić and A. M. Fink, Classical and New Inequalities in Analysis, Mathematics and its Applications (East European Series), 61. Kluwer Academic Publishers Group, Dordrecht, 1993.

[13] J. Pečarić and R. Rajić, The Dunkl-Williams inequality with $n$ elements in normed linear spaces, Math. Inequal. \& Appl., 10(2007), 461-470.

[14] J. Pečarić, F. Proschan and Y. L. Tong, Convex Functions, Partial Orderings, and Statistical Applications. Mathematics in Science and Engineering, 187. Academic Press, Inc., Boston, MA, 1992.

School of Engineering and Science, Victoria University, PO Box 14428, Melbourne VIC 8001, Australia.

E-mail: sever.dragomir@vu.edu.au 